Research Article

\section{A study on pacemaker pocket infection}

\section{Goutam Datta*}

Associate Professor, Burdwan Medical College, India

\section{Abstract}

Objective: Cardiac implantable electronic device (CIED) infections now constitute $\sim 10 \%$ of all endocarditis cases. The incidence of CIED infection is usually $<2 \%$. Our objective was to study pacemaker pocket infection rate and different risk factors in our institution.

Methods: This observational study was conducted over a period of five years from January 2011 to December 2016 and it included 1096 patients. Common risk factors like patients with diabetes, repeat procedure, chronic renal failure, chronic obstructive airway disease, immunosuppressive agents were studied in our patients.

Results: Our study consisted of 1096 patients. Pacemaker pocket infection occurred in sixteen patients $(1.5 \%)$. Chronic renal failure patients were one hundred thirty in our study $(11.86 \%)$. There were three hundred fifty six diabetic patients $(32.48 \%)$. Repeat procedure was done in ninety five patients (8.6\%).

Eighty six patients were suffering from chronic obstructive airway (7.8\%). Patients on immunosuppressive therapy were fourteen in our study (1.2\%).

Conclusion: Pacemaker pocket infections is a dreaded complication after pacemaker implantation. During implantation, there is a risk of device contamination with the patient's own skin flora and it can be prevented by ideal surgical asepsis technique, pre and perioperative use of antibiotics.

\section{More Information}

*Address for Correspondence: Goutam Datta, MD.DNB.DM.MRCP(UK), Associate Professor, Burdwan Medical College, P 3 Lake Gardens Govt. Housing, 48/4 Sultan Alam Road, Calcutta -33, India, Tel: 919433415086 ; Email: goutamdattadn@yahoo.in

Submitted: 10 March 2020

Approved: 22 March 2020

Published: 25 March 2020

How to cite this article: Datta G. A study on pacemaker pocket infection. J Cardiol Cardiovasc Med. 2020; 5: 056-059.

DOI: 10.29328/journal.jccm.1001087

Copyright: ( $) 2020$ Datta G. This is an open access article distributed under the Creative Commons Attribution License, which permits unrestricted use, distribution, and reproduction in any medium, provided the original work is properly cited.

Keywords: Cardiac pacemaker; Pacemaker pocket infection; Different risk factors

Check for updates

OPEN ACCESS

\section{Introduction}

Permanent pacemaker was introduced in clinical practice since 1960s. Incidence of pacemaker pocket infection varies between 1\% - 12.5\% [1]. Lead dislodgement, vascular injury, pocket hematoma and pocket infection are common short term complications after permanent pacemaker implantation. Long term complications include lead fracture, insulation failure, premature battery depletion and pocket infection. Pacemakers have both intravascular and extravascular components.

Infection can occur in pacemaker pocket, pacing leads and native cardiac structures or various combinations. Several factors are implicated for pocket infection. Our objective was to study incidence and different risk factors of pacemaker pocket infection.

\section{Methods}

Our centre is a university medical college hospital covering five districts of our state. One thousand and ninety six patients were studied over a period of five years from January 2011 to December 2016. Informed consents were taken from all patients and the study protocol conforms to the ethical guidelines of the 1975 Declaration of Helsinki with prior approval by institution's human research committee. This was an observational study. CRT and ICD patients were not selected in our study. Last patient was enrolled in January 2015. Minimum follow up was one year and maximum was six years. Patients with age more than eighteen years were included in our study. Common risk factors like diabetes, repeat procedure, chronic renal failure, chronic obstructive airway disease, immunosuppressive agents were studied. Three layers closure technique were followed for skin closure.

Non absorbable monofilament suture material had been used for lead fixation and absorbable suture material were used for skin closure. Pre and perioperative antibiotics were also given in every case. Amoxycillin/Clavulanic acid and Linezolid were used for five days as antibiotic protocol.

\section{Statistical methods}

For statistical analysis, Med Calc software for windows was used to compare the incidence rate and prevalence of different risk factors. 


\section{Results}

Majority of our implantations were single chamber pacemaker because of economic reasons. One thousand and fifty four $(70 \%)$ implantations were single chamber pacemaker and six hundred sixty (30\%) were double chamber pacemaker. There were one thousand five hundred eighty four (72\%) male patients and six hundred sixty (28\%) female patients (Table $1)$. There were two hundred and eighteen patients in twenty to fifty years age group (19.8\%).

Seven hundred and ninety one patients (72\%) were in fifty to eighty years age group and eighty seven patients $(7.93 \%)$ were in more than eighty years age group.

Sub pectoral pockets were done in two hundred twenty patients $(10 \%)$ of cases. It was necessary in patients with thin built stature. Incidence of pocket haematoma as well pocket infection were more in this group.

Thirty patients had pocket haematoma in patients with sub pectoral pockets (13.9\%). Two patients had pocket infection in this group (.9\%).

Many patients were suffering from different associated diseases in our study. Chronic renal failure patients were one hundred thirty in our study (11.5\%). There were three hundred fifty six diabetic patients (32\%). Repeat procedure was done in ninety five patients (9\%) (Table 2). Prolonged procedural time is usually associated with increased risk of infection but we did not find such correlation in our study.

Eighty six patients were suffering from chronic obstructive airway (7\%). Patients on immunosuppressive therapy were fourteen in our study (1.09\%). Fifty four patients had pacemaker pocket haematoma (4.9\%).

Pacemaker pocket infection occurred in sixteen patients. Incidence of pacemaker pocket infection in our study was

\begin{tabular}{|c|c|c|}
\hline \multicolumn{3}{|c|}{ Table 1: Different population characteristics. } \\
\hline Category of sample & Study group & \% study group \\
\hline Male & 789 & 72 \\
\hline Female & 307 & 28 \\
\hline Single chamber pacemaker & 760 & 69 \\
\hline Double chamber pacemaker & 336 & 31 \\
\hline Age(20-50) & 218 & 19.8 \\
\hline Age (50-80) & 791 & 72 \\
\hline Age $>80$ & 87 & 7.93 \\
\hline
\end{tabular}

Table 2: Different risk factors and comparison of infection rate for each risk factors.

\begin{tabular}{|c|c|c|c|c|}
\hline \multicolumn{1}{|c|}{ Risk factors } & Frequency & (\%) & No of infection & $\begin{array}{c}\text { \% of Infection amongst } \\
\text { risk factors }\end{array}$ \\
\hline Diabetes & 356 & 32.48 & 4 & 16.18 \\
\hline Chronic renal failure & 130 & 11.86 & 3 & 5.9 \\
\hline $\begin{array}{c}\text { Chronic obstructive } \\
\text { airway disease }\end{array}$ & 86 & 7.8 & 2 & 7.8 \\
\hline $\begin{array}{c}\text { Repeat procedures } \\
\text { Immunosuppressive }\end{array}$ & 95 & 8.6 & 6 & 27.74 \\
\hline \begin{tabular}{c} 
Therapy \\
\hline
\end{tabular} & 14 & 1.2 & 1 & 1.2 \\
\hline
\end{tabular}

1.5\%. Lead endocarditis were seen in six patients. Four patients had associated pacemaker pocket infection. Two patients did not have associated pocket infection.

According to our institutional protocol all patients after explanation were kept on parenteral antibiotic therapy for two weeks. Antibiotic regimen comprises of Vancomycin and gentamicin.

Incidence of infection according to time frame (Table 3):

Infection rate in different subgroups were shown in table 4.

\section{Discussion}

Permanent pacemakers have been used for over 50 years. Infection is a serious complication of cardiac device implantation and is associated with increased morbidity, mortality, and healthcare costs. In- hospital mortality rates have been reported to be $3.7 \%-11.3 \%$ [2]. The standard of-care treatment requires device removal and systemic antibiotic therapy. The additional admission costs of an infected device can exceed \$15 000 in the USA and $€ 7000$ in Europe [3]. An analysis of the Nationwide Inpatient Sample discharge records from 1993 through 2008 showed a 96\% increase in Cardiac implantable electronic device (CIED) implantations in the USA. During the same period, the incidence of CIED infection increased by $210 \%$ (from $1.5 \%$ in 1993 to $2.4 \%$ in 2008) [4]. Pacemaker pocket infection rate varies between $1 \%-12.5 \%$ [4]. In our institution, infection rate was $1.5 \%$. Multicenter U.S. and European data from the MOST (Mode Selection in Sinus Node Dysfunction Trial) and FOLLOWPACE (Cost-Effectiveness of Routine Follow-up Visits in Patients With a Pacemaker) studies report 30 - and 60day complication rates of $4.8 \%$ and $12.4 \%$, respectively, and 3 years and 5 years complication rates of $7.5 \%$ and $19.7 \%$, respectively.

Short term complication of single and dual chamber pacemakers include lead revision (2.5\% and $3.7 \%)$, infection ( $1.2 \%$ and $1.1 \%)$, cardiac perforation $(0.3 \%$ and $0.6 \%)$, venous thrombosis $(0.4 \%$ and $0.5 \%)$, pocket complications $(0.3 \%$ and $0.3 \%)$, and generator complications $(0.1 \%$ and $0.1 \%)$.

\begin{tabular}{|c|c|c|}
\hline \multicolumn{2}{|l|}{ Table 3} \\
\hline No of months after implantation & No of infection & (\%) \\
\hline Less than two months & 1 & 6.25 \\
\hline Two to six months & 5 & 31.25 \\
\hline Six to twelve months & 8 & $50 \%$ \\
\hline More than twelve months & 2 & 12.5 \\
\hline
\end{tabular}

\begin{tabular}{|c|c|}
\hline Table 4: Different types of infection in study group. \\
\hline Types of infection & Study group \\
\hline Type 1 & 4 \\
\hline Type 2 & 3 \\
\hline Type 3 & 5 \\
\hline Type 4 & 3 \\
\hline Type 5 & 1 \\
\hline
\end{tabular}


Lead removals, which comprise $1.4 \%$ of all lead- related complications, were associated with infection in $22.9 \%$ of the cases [6]. Permanent pacemakers are implanted commonly through cephalic vein or subclavian vein, rarely via jugular or axillary vein. Pulse generator is kept in pacemaker pocket which requires extensive fascial plane dissection. Dissected tissue and skin should be closed properly for better wound healing. Wound contamination by microorganism is the root cause of early or primary infection. Secondary infection may be caused by wound or device seeding by blood borne organisms [7]. Early El Cajone and University of Oklahoma experience of combination of prophylactic systemic antibiotics and topical pocket irrigation with povidone-iodine has been reported useful in the prevention of infection [8]. Early infections after pacemaker implantation are thought to result from wound contamination at the time of surgery and most of these seem to happen within the first sixty days after the implantation.

Two thousand five hundred and sixty four patients were studied by Dhanunjaya Lakkireddy, el al. over a period of eight years who had either new device implantation and or lead/ generator replacement. Of these patients, 1,359 (53\%) had their pockets irrigated with povidone-iodine solution versus 1,205 (47\%) with saline, prior to device placement. Eighteen patients $(0.7 \%)$ developed pocket infection [9]. In their series 33\% had diabetes, 5\% had renal insufficiency, 7\% had autoimmune disorders, and $17 \%$ were on systemic steroids. In our series $11.5 \%$ had chronic renal failure, $32 \%$ had diabetes, $9 \%$ had repeat procedure, $7 \%$ had chronic obstructive airway disease and $1.2 \%$ patients were on immune suppressive therapy. Pacemaker pocket infection occurred in 33 patients $(1.5 \%)$ in our series.

Pacemaker pocket infection is characterized by localized erythema, cellulitis, swelling or pain over the pocket. It may progress to wound dehiscence, purulent discharge, skin erosion or sinus formation. There are different classification of pacemaker pocket infection.

Type 1) Spreading cellulitis affecting the generator site.

Type 2) Incision site purulent exudate (excluding simple stitch abscess).

Type 3) wound dehiscence.

Type 4) erosion through skin with exposure of the generator or leads.

Type 5) abscess or fistula formation.

Complicated pocket infection is associated with evidence of lead or endocardial involvement, systemic signs or symptoms of infection or positive blood cultures. Gram-positive bacteria were by far the most commonly isolated microorganisms (67\%). Coagulase negative staphylococci is the most consistently isolated bacteria followed closely by Staphylococcus aureus. Gram-negative bacilli were isolated in $1 \%-17 \%$ of patient episodes. Fungal infection is uncommon, occurring in no more than $2 \%$ of patients [10]. Pocket infections occurred late or a full year after pocket manipulation in $50 \%$ of cases in a study by Sohail, et al. and most were due to coagulase-negative staphylococci (CoNS). Unlike infections due to staphylococcus aureus, infections due to CoNS are more indolent and present with subtler findings. This suggests that CoNS infections were likely acquired during pocket intervention [11]. A variety of patient characteristics and procedural issues have been associated with pacemaker infections. Konstantinos, et al. have described several risk factors for infection in their study [12]. Regarding host-related factors, the most significant predictors of infection are diabetes mellitus (OR 2.08), end-stage renal disease (OR 8.73), COPD (OR 2.95), corticosteroid drug use (OR 3.44), history of previous device infection (OR 7.84), renal insufficiency (OR 3.02), malignancy (OR 2.23) and congestive heart failure (OR 1.65). Other significant host factors include New York Heart Association (NYHA) functional class $\geq 2$, fever prior to implantation, oral anticoagulation, heparin bridging, and chronic skin disorders. Regarding procedure-related factors, post-operative haematoma (OR 8.46), reintervention for lead dislodgement (OR 6.37), device replacement/revision (OR 1.98), lack of antibiotic prophylaxis (OR 0.32), temporary pacing (OR 2.31), generator change (OR 1.74), inexperienced operator (OR 2.85) and increased procedure duration were all significant predictors of CIED infection. Among device related characteristics, abdominal generator pocket (OR 4.01), the presence of epicardial leads (OR 8.09), positioning of two or more leads (OR 2.02), and dual-chamber system (OR 1.45) were predictors of CIED infection. Post-operative haematoma has been repeatedly associated with the risk for CIED infections and was a strong predictor of infection in their analysis.

\section{Conclusion}

Pacemaker pocket infections is a dreaded complication after pacemaker implantation. During implantation, there is a risk of device contamination with the patient's own skin flora, introduced into the wound at the time of skin incision and it can be prevented by ideal surgical asepsis technique, pre and perioperative use of antibiotics.

\section{References}

1. Nery PB, Fernandes R, Nair GM, Sumner GL, Ribas CS, et al. Devicerelated infection among patients with pacemakers and implantable defibrillators: incidence, risk factors, and consequences. J Cardiovasc Electrophysiol. 2010: 21, 786- 90.

PubMed: https://www.ncbi.nlm.nih.gov/pubmed/20102431

2. Cantillon DJ, Exner DV, Badie N, Davis K, Gu NY, et al. Complications and Health Care Costs Associated With Transvenous Cardiac Pacemakers in a Nationwide Assessment. JACC: Clinical Electrophysiology. 2017; 3: 1296-1305.

PubMed: https://www.ncbi.nlm.nih.gov/pubmed/29759627

3. Poole JE, Gleva MJ, Mela T, Chung MK, Uslan DZ, et al. Complication rates associated with pacemaker or implantable cardioverterdefibrillator generator replacements and upgrade procedures: results 
from the REPLACE registry. Circulation. 2010; 122:1553-1561.

PubMed: https://www.ncbi.nlm.nih.gov/pubmed/20921437

4. Margey R, McCann H, Blake G, Keelan E, Galvin J, et al .Contemporary management of and outcomes from cardiac device related infections. Europace. 2010; 12: 64 -70.

PubMed: https://www.ncbi.nlm.nih.gov/pubmed/19910314

5. Sohail MR, Henrikson CA, Braid Forbes MJ, Forbes KF, Lerner DJ. Mortality and cost associated with cardiovascular implantable electronic device infections. Arch Intern Med. 2011; 171: 182-186. PubMed: https://www.ncbi.nlm.nih.gov/pubmed/21911623

6. Greenspon AJ, Patel JD, Lau E, Ochoa JA, Frisch DR, et al. 16-year trends in the infection burden for pacemakers and implantable cardioverter-defibrillators in the United States 1993 to 2008. J Am Coll Cardiol. 2011; 58: 1001-1006.

PubMed: https://www.ncbi.nlm.nih.gov/pubmed/21867833

7. Lee I, Agarwal RK, Lee BY, Fishman NO, Umscheid CA. Systematic review and cost analysis comparing use of chlorhexidine with use of iodine for preoperative skin antisepsis to prevent surgical site infection. Infect Control Hosp Epidemiol. 2010; 31: 1219- 1229. PubMed: https://www.ncbi.nlm.nih.gov/pubmed/20969449

8. Bertaglia E, Zerbo F, Zardo S, Barzan D, Zoppo F, et al. Antibiotic prophylaxis with a single dose of cefazolin during pacemaker implantation: incidence of long-term infective complications. Pacing Clin Electrophysiol. 2006; 29: 29-33.

PubMed: https://www.ncbi.nlm.nih.gov/pubmed/16441714

9. Lakkireddy D, Valasareddi S, Ryschon K, Basarkodu K, Rovang K, et al The impact of povidone-iodine pocket irrigation use on pacemaker and defibrillator infections. Pacing Clin Electrophysiol. 2005; 28: 789- 794. PubMed: https://www.ncbi.nlm.nih.gov/pubmed/16105006

10. Athan $E$, Chu VH, Tattevin $P$, Selton-Suty $C$, Jones $P$, et al. Clinical characteristics and outcome of infective endocarditis involving implantable cardiac devices. JAMA. 2012; 307: 1727- 1735.

PubMed: https://www.ncbi.nlm.nih.gov/pubmed/22535857

11. Darouiche R, Mosier M, Voigt J. Antibiotics and antiseptics to prevent infection in cardiac rhythm management device implantation surgery. Pacing and Clinical Electrophysiology. 2012; 35: 1348- 1360. PubMed: https://www.ncbi.nlm.nih.gov/pubmed/22946683

12. Konstantinos A, Polyzos Athanasios A, Konstantelias Matthew E. Falagas. Risk factors for cardiac implantable electronic device infection: a systematic review and meta-analysis. Europace. 2015; 17: 767-767.

PubMed: https://www.ncbi.nlm.nih.gov/pubmed/25926473 